\title{
Discovery of the optical counterpart to the X-ray pulsar SAX J2103.5+4545
}

\author{
P. Reig ${ }^{1,4}$, I. Negueruela ${ }^{2}$, J. Fabregat ${ }^{3}$, R. Chato $^{1}$, P. Blay ${ }^{1}$, and F. Mavromatakis ${ }^{4}$ \\ ${ }^{1}$ GACE, Instituto de Ciencias de los Materiales, Universitad de Valencia, 46071 Paterna-Valencia, Spain \\ e-mail: [pere.blay; rachid.chato]@uv.es \\ 2 Departamento de Física, Ingeniería de Sistemas y Teoría de la Señal, Universidad de Alicante, 03080 Alicante, Spain \\ e-mail: ignacio@dfists.ua.es \\ 3 Observatorio Astronómico de Valencia, Universitad de Valencia, 46071 Paterna-Valencia, Spain \\ e-mail: juan. fabregat@uv.es \\ ${ }^{4}$ University of Crete, Physics Department, PO Box 2208, 71003 Heraklion, Crete, Greece \\ e-mail: fotis@physics.uoc.gr
}

Received 2 December 2003 / Accepted 26 March 2004

\begin{abstract}
We report optical and infrared photometric and spectroscopic observations that identify the counterpart to the 358.6-s X-ray transient pulsar SAX J2103.5+4545 with a moderately reddened $V=14.2$ B0Ve star. This identification makes SAX J2103.5+4545 the Be/X-ray binary with the shortest orbital period known, $P_{\text {orb }}=12.7$ days. The amount of absorption to the system has been estimated to be $A_{\mathrm{V}}=4.2 \pm 0.3$, which for such an early-type star implies a distance of about $6.5 \mathrm{kpc}$. The optical spectra reveal major and rapid changes in the strength and shape of the $\mathrm{H} \alpha$ line. The $\mathrm{H} \alpha$ line was initially observed as a double peak profile with the ratio of the intensities of the blue over the red peak greater than one $(V / R>1)$. Two weeks later this ratio reversed $(V / R<1)$. Subsequently, in less than a month, the emission ceased and $\mathrm{H} \alpha$ appeared in absorption. This fast spectral variability is interpreted within the viscous decretion disc model and demonstrates the significant role of the neutron star on the evolution of the circumstellar disc around the Be star. The implications of the small orbit and moderate eccentricity on the spin period of the neutron star are discussed.
\end{abstract}

Key words. stars: individual: SAX J2103.5+4545 - X-rays: binaries - stars: neutron - stars: binaries: close stars: emission-line, Be

\section{Introduction}

The X-ray transient SAX J2103.5+4545 was discovered by the BeppoSAX Wide Field Camera (WFC) during an outburst in February 1997 (Hulleman et al. 1998). The source was active for about eight months and reached a peak intensity of $20 \mathrm{mCrab}(2-25 \mathrm{keV})$ on April 11, 1997. The X-ray flux showed pulsed emission with a period of $358.61 \mathrm{~s}$. An absorbed power law $\left(\mathrm{d} N / \mathrm{d} E \propto \mathrm{e}^{-N_{\mathrm{H}} \sigma(E)} E^{-\Gamma}\right)$ with photon number index $\Gamma=1.27$ and column density $N_{\mathrm{H}}=3.1 \times 10^{22} \mathrm{~cm}^{-2}$ was used to fit the X-ray energy spectrum.

A second outburst was detected on October 25, 1999 by the All Sky Monitor on board RXTE (Baykal et al. 2000), reaching a peak intensity of $27 \mathrm{mCrab}(2-12 \mathrm{keV})$ three days later. The longer duration of this activity period (about 14 months) and regular monitoring allowed a Doppler shifts analysis of the pulsations resulting in the derivation of the orbital parameters (Baykal et al. 2000): the system has a moderately eccentric orbit, $e=0.4 \pm 0.2$, and an orbital period of $12.68 \pm 0.25$ days.

Send offprint requests to: $\mathrm{P}$. Reig, e-mail: pablo.reig@uv.es
The RXTE 3-50 keV X-ray energy spectrum was well described by an absorbed power law, modified by a cutoff at around $8 \mathrm{keV}$. In addition, the fits required an emission iron line at $6.4 \mathrm{keV}$. The power-law index and the flux of the iron line were seen to vary with X-ray intensity (Baykal et al. 2002).

Inam et al. (2004) reported the detection of a $0.044 \mathrm{~Hz}$ QPO using XMM-Newton data, and estimated the magnetic field of the neutron star to be $\sim 7 \times 10^{12} \mathrm{G}$. These authors also found that the energy spectrum at low energies required a soft component with a characteristic temperature of $1.9 \mathrm{keV}$ (if modeled as a blackbody).

The nature of the optical counterpart to SAX J2103.5+4545 was uncertain. The shape of the X-ray energy spectrum, the transient behaviour and the increase of the X-ray intensity around periastron passages (Baykal et al. 2002) point toward a Be/X-ray binary. However, SAX J2103.5+4545 does not follow the correlation between the orbital period and the spin period (Corbet 1986) of other Be/X-ray systems. Although Hulleman et al. (1998) tentatively suggested that the star HD 200709 might be linked to SAX J2103.5+4545, its position outside the BeppoSAX error box $\left(\sim 2^{\prime}\right)$ and its late spectral 
Table 1. Optical and infrared magnitudes of SAX J2103.5+4545.

\begin{tabular}{lcccc}
\hline \hline \multicolumn{5}{c}{ Optical } \\
\hline Date & $B$ & $V$ & $R$ & $I$ \\
\hline $08 / 06 / 03$ & $15.34 \pm 0.02$ & $14.22 \pm 0.02$ & $13.48 \pm 0.02$ & - \\
$24 / 08 / 03$ & $15.36 \pm 0.03$ & $14.27 \pm 0.02$ & $13.59 \pm 0.03$ & $12.87 \pm 0.03$ \\
\hline \multicolumn{5}{c}{ Infrared } \\
\hline Date & $J$ & $K$ \\
\hline $24 / 08 / 03$ & $11.97 \pm 0.03$ & $11.42 \pm 0.03$ & $11.20 \pm 0.03$ \\
$27 / 08 / 03$ & $11.38 \pm 0.03$ & $10.90 \pm 0.03$ & $10.67 \pm 0.03$ \\
$05 / 12 / 03$ & $11.41 \pm 0.03$ & $10.97 \pm 0.03$ & $10.75 \pm 0.03$ \\
$04 / 01 / 04$ & $11.40 \pm 0.04$ & $10.96 \pm 0.05$ & $10.75 \pm 0.04$ \\
$07 / 01 / 04$ & $11.40 \pm 0.04$ & $10.94 \pm 0.04$ & $10.71 \pm 0.03$ \\
\hline
\end{tabular}

type (B8V) posed a serious hindrance to its candidacy as the correct optical counterpart.

In this work we present ground-based observations that identify the optical counterpart to SAX J2103.5+4545 and classify it as a Be/X-ray binary. The fast optical spectral variability, $\mathrm{X}$-ray behaviour and position in the $P_{\text {spin }}-P_{\text {orb }}$ diagram are explained within the viscous decretion disc model.

\section{Observations}

\subsection{Optical observations}

The field around the best-fit X-ray position given by the BeppoSAX Wide Field Camera was observed through the Johnson $B, V$, and $R$ filters from the $1.3 \mathrm{~m}$ telescope of the Skinakas observatory on June 8, 2003 and August 24, 2003. using a $1024 \times 1024$ SITe CCD chip with a $24 \mu \mathrm{m}$ pixel size (corresponding to $0.5^{\prime \prime}$ on sky). On June 8, 2003 the field was also observed through an interference filter centred at $6563 \AA$, and a width of $10 \AA$ (H $\alpha$ filter). Standard stars from the Landolt list (Landolt 1992) were used for the transformation equations. Reduction of the data was carried out in the standard way using the IRAF tools for aperture photometry. The photometric magnitudes are given in Table 1 . Figure 1 shows a $V$-band image of the field of SAX J2103.5+4545. The $\sim 2^{\prime}$ BeppoSAX WFC (Hulleman et al. 1998), 1.2' INTEGRAL ISGRI and $~ 30^{\prime \prime}$ INTEGRAL JEM-X (Blay et al. 2004) error circles are also shown.

Optical spectroscopic observations were obtained from the Skinakas observatory in Crete (Greece) on August 1, 2003 and October 6 and 8, 2003 and from the observatory of El Roque de los Muchachos in La Palma (Spain) on the night August 17, 2003. The $1.3 \mathrm{~m}$ telescope of the Skinakas Observatory was equipped with a $2000 \times 800$ ISA SITe CCD and a $13021 \mathrm{~mm}^{-1}$ grating, giving a nominal dispersion of $\sim 1 \AA$ Apixel. The William Herschel $4.2 \mathrm{~m}$ telescope (WHT) was equipped with the ISIS double-armed (R1200B and R1200R gratings) spectrograph, giving a dispersion of $0.23 \AA /$ pixel. The blue (3900-4700 $\AA$ ) and red (6100-6900 $\AA$ ) spectra were obtained with the $4096 \times$ $2048(13.5 \mu \mathrm{m})$ pixels EEV12 CCD and MARCONI2 CCD,

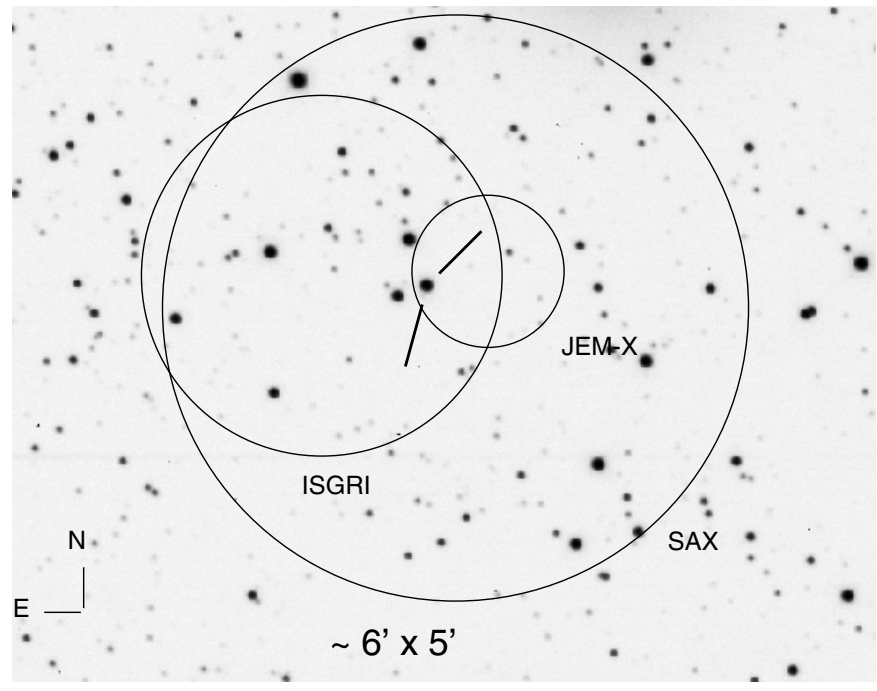

Fig. 1. Optical $V$ filter image of the field around SAX J2103.5+4545, with the X-ray position uncertainty circles from the WFC onboard BeppoSAX (99\% confidence level) and ISGRI and JEM-X onboard INTEGRAL ( $90 \%$ confidence level). The coordinates of the proposed optical counterpart are RA $=21: 03: 35.7 \mathrm{Dec}=+45: 45: 04$ (Eq. 2000).

respectively. Further spectra were obtained through the service time scheme from the WHT on the night of September 14, 2003. The instrumental set-up was the same as above except for the fact that lower resolution gratings (R600B and R600R) were employed, providing a dispersion of $0.44 \AA /$ pixel but a larger wavelength coverage.

The reduction of the spectra was made using the STARLINK Figaro package (Shortridge et al. 2001), while their analysis was performed using the STARLINK Dipso package (Howarth et al. 1998).

\subsection{Infrared observations}

Infrared photometry in the $J H K$ bands was done at the $1.5 \mathrm{~m}$. Carlos Sánchez Telescope (TCS) located at the Teide Observatory in Tenerife, Spain. The instrument used was the Cain-II camera, equiped with a $256 \times 256 \mathrm{HgCdTe}$ (NICMOS 3 ) detector. Instrumental magnitudes were obtained from the images by means of the IRAF tools for aperture photometry. Instrumental values were transformed to the standard system defined by the standard star list published by Hunt et al. (1998). The accuracy of the standard values, defined as the standard deviation of the mean catalogue minus transformed values for the standard stars, is 0.03 mag. in all three bands. The obtained values are given in Table 1. An important variation of about 0.5 mag. in all IR bands from 24 to 27 August is noticeable. This is in contrast with the small variation from 27 August to 05 December, and also with the small variations in the optical bands. To check the reliability of the data we have obtained photometry of seven nearby stars present in the same images. All of them appear constant in all bands, within the accuracy of the photometry. Hence we can safely exclude the possibility of any instrumental effect, and confirm that in August 24 SAX J2103.5+4545 was significantly fainter in all IR bands. 


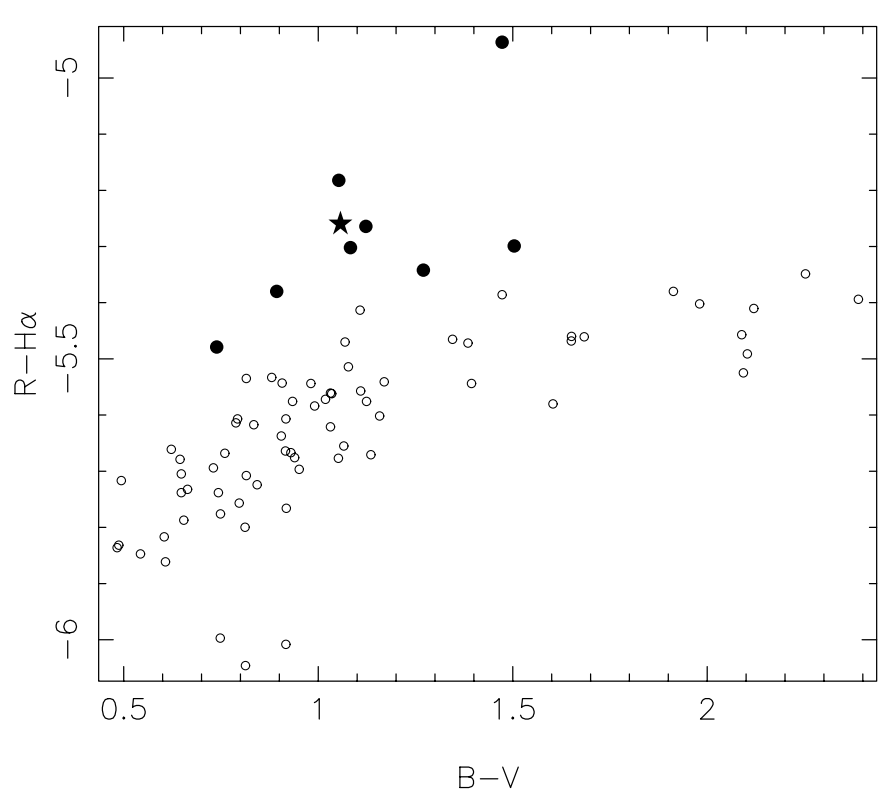

Fig. 2. Colour-colour diagram of the field around SAX J2103.5+4545. The star symbol marks the position of the proposed optical counterpart. Filled-circles represent strong $\mathrm{H} \alpha$ emitters, while open circles correspond to field stars.

\section{Results}

\subsection{The colour-colour diagram}

The instrumental magnitudes corresponding to the $B, V, R$ and $\mathrm{H} \alpha$ filters obtained during the June 8, 2003 observations were used to define a "blue" colour $(B-V)$ and a "red" colour $(\mathrm{R}-\mathrm{H} \alpha)$. Then a colour-colour diagram was constructed by plotting the red colour as a function of the blue colour as shown in Fig. 2. Stars with a moderately or large $\mathrm{H} \alpha$ excess can be distinguished from the rest because they deviate from the general trend and occupy the upper parts of the diagram (filled circles in Fig. 2). This kind of diagrams have been successfully used to identify optical counterparts in the Magellanic Clouds (Grebel 1997; Stevens et al. 1999). Of the approximately 80 stars that were analysed in the $\sim 8^{\prime} \times 8^{\prime}$ field of view of the $1.3 \mathrm{~m}$ telescope about 8 were bright enough in $\mathrm{H} \alpha$ to call our attention. The position of each one of these potential candidates with respect to the satellites error circle was then checked. Of these relatively strong $\mathrm{H} \alpha$ emitters only one lied inside the WFC BeppoSAX satellite position uncertainty circles (Fig. 1). As well as appearing bright in $\mathrm{H} \alpha$, this star is highly reddened, $(B-V)>1$, making a good case for a Be classification. However, the photometric data are not conclusive since other type of systems, such as supergiants or late-type giants can also show emission lines and/or have a very reddened spectrum. The final confirmation that the system contains an early-type star was provided by the spectroscopic observations, which also revealed $\mathrm{H} \alpha$ in emission (Fig. 4). Even more significant than the emission itself it is the double-peak profile, since it can be interpreted as coming from the circumstellar envelope of a Be star.

\subsection{Spectral type}

A visual comparison of the SAX J2103.5+4545 spectrum (Fig. 3) with those of MK standards in the Walborn \& Fitzpatrick (1990) atlas reveals that the ratios He I $\lambda 4026 / \mathrm{He} \mathrm{II} \lambda 4200$ and He I $\lambda 4713 / \mathrm{He}$ II $\lambda 4686$ are very similar to those of the standard star $v$ Ori (B0V). Indeed, the presence of He II $(\lambda 4200, \lambda 4686)$ indicates that the spectral type of the optical star is earlier than B1. Specifically, He II $\lambda 4686$ is last seen at B0.5-B0.7. On the other hand, the weakness of He II $\lambda 4541$ relative to He I $\lambda 4471$ indicates an spectral type later than $\mathrm{O} 9$.

With regard to the luminosity classification, the weakness of Si III $\lambda 4552-68$ and the fact that the line intensitie of He II $\lambda 4686$ is larger than that of He I $\lambda 4713$ seem to indicate a main-sequence star. Other luminosity indicators are the ratios of He lines to nearby metallic lines. SAX J2103.5+4545 shows He II $\lambda 4686 /$ CIII $\lambda 4650 \sim 1$ and He I $\lambda 4144 /$ Si IV $4089 \sim 1$. A main-sequence star would have those ratios $\sim 1$ and $\gtrsim 1$, respectively. Therefore, we conclude that the optical counterpart to the X-ray accreting pulsar SAX J2103.5+4545 is a B0V star, although a subgiant cannot be excluded. This classification puts SAX J2103.5+4545 in the group of Be/X-ray binaries.

\subsection{Evolution of $\mathrm{H} \alpha$}

Profile changes in emission lines, particularly, $\mathrm{H} \alpha$, can be used to trace the dynamical evolution of the Be envelope (Negueruela et al. 2001). The $\mathrm{H} \alpha$ profile obtained from each observing run is presented in Fig. 4. The spectra revealed a highly variable $\mathrm{H} \alpha$ emission line. Table 2 gives the measurements of the equivalent width of the $\mathrm{H} \alpha$ line for each observing run. Negative values indicate that the line is in emission. In about two weeks the ratio of the blue to the red peak reversed and the strength of the $\mathrm{H} \alpha$ line decreased by $~ 50 \%$. The peak separation, however, remained constant at about $440 \mathrm{~km} \mathrm{~s}^{-1}$. The line profile of the August 17, 2003 spectrum is reminiscent of the "shell class", i.e., a double peak emission with central absorption presumably due to self-absorption as a consequence of a high inclination angle. Subsequently, in less than a month, the line appears in absorption. A very small amount of remaining emission might still be present in the September 14, 2003 spectrum. For a normal absorption-line B0V star the $\mathrm{H} \alpha$ equivalent width is approximately $+3.5 \AA$ (Table 9.1 in Jaschek \& Jaschek 1987). The lastest spectra seem to indicate that a new emission phase has begun. The October 8, 2003 spectrum shows incipient emission at the bottom of the absorption profile.

\subsection{Reddening and distance}

Besides the $\mathrm{H} \alpha$ and $\mathrm{He} \mathrm{I} \lambda 6678$ lines, the red spectrum (Fig. 5) contains several strong diffuse interstellar bands (DIB), which can be used to estimate the amount of interstellar absorption toward the source (Herbig 1975; Herbig \& Leka 1991; Galazutdinov et al. 2000). Table 3 summarises the measurements of the equivalent width and the corresponding estimated value of the colour excess $E(B-V)$ according to the linear relations of Herbig (1975) for the WHT observations. The quoted 


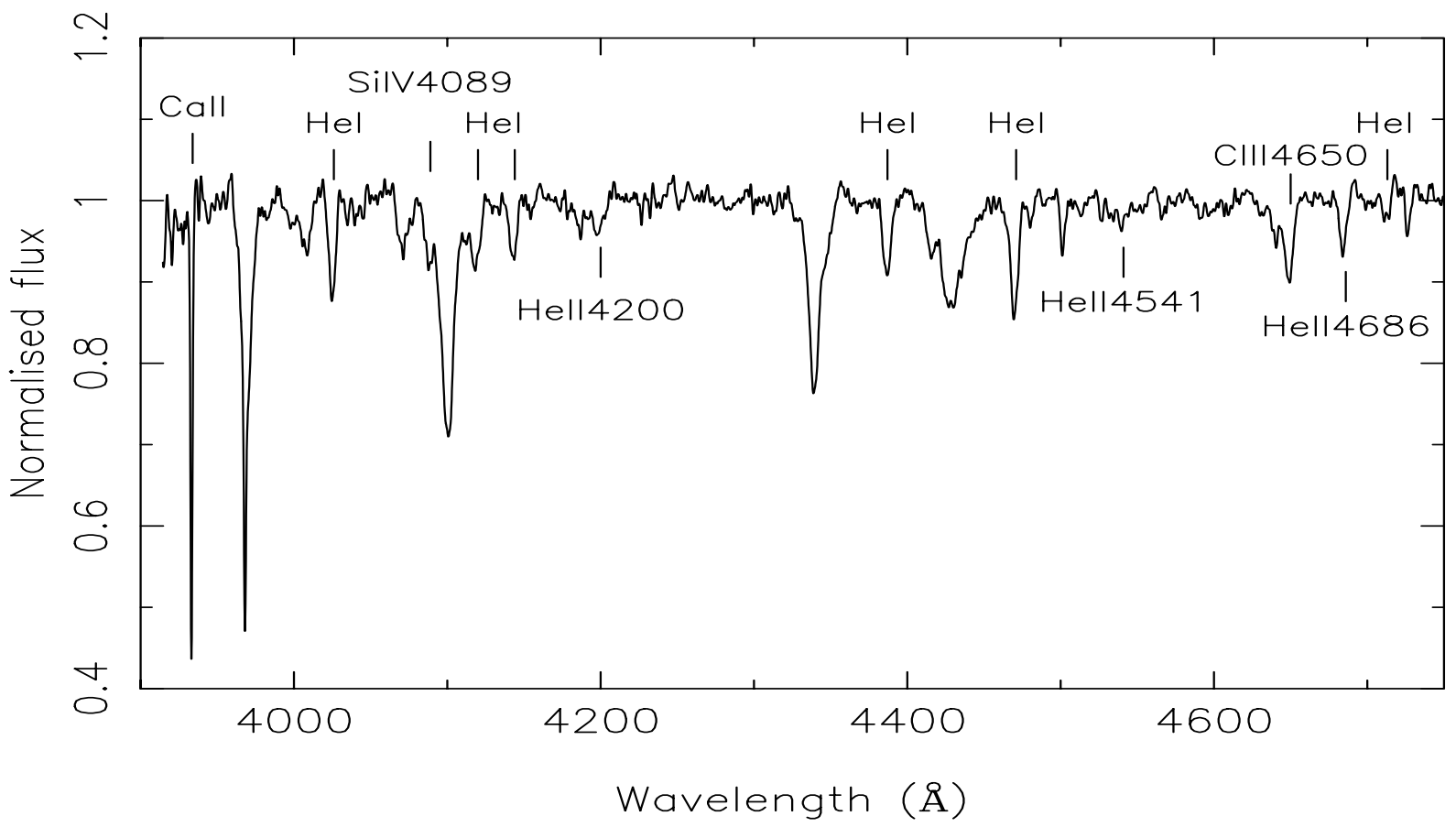

Fig. 3. Blue spectrum showing the lines used for spectral classification. The spectrum was taken from the WHT on August $17,2003$. The He I lines marked are $\lambda 4026, \lambda 4121, \lambda 4144, \lambda 4387, \lambda 4471, \lambda 4713$. Also shown is the insterstellar line Ca II $\lambda 3934$.

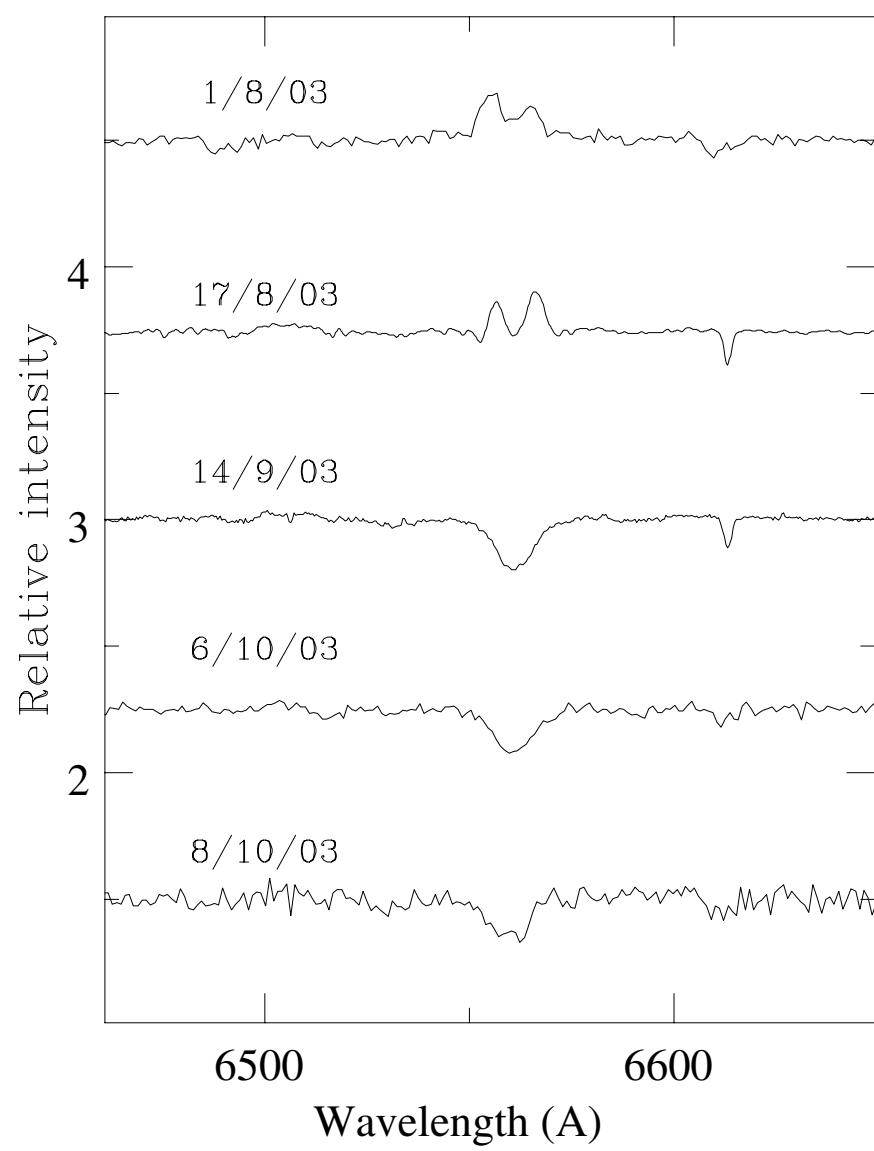

Fig. 4. Evolution of the $\mathrm{H} \alpha$ line.

values for the September 14, 2003 spectra are the average of six measurements. Those of August 17, 2003 correspond to one
Table 2. H $\alpha$ equivalent width measurements. Errors are $\leq 10 \%$.

\begin{tabular}{cccc}
\hline \hline Date & MJD & $\begin{array}{c}E W(\mathrm{H} \alpha) \\
(\AA)\end{array}$ & Telescope \\
\hline $01 / 08 / 03$ & 52853 & -2.20 & SKI \\
$17 / 08 / 03$ & 52869 & -1.07 & WHT \\
$14 / 09 / 03$ & 52897 & +2.32 & WHT \\
$06 / 10 / 03$ & 52919 & +2.15 & SKI \\
$08 / 10 / 03$ & 52921 & +1.95 & SKI \\
\hline
\end{tabular}

spectrum, with higher spectral resolution. The colour excess estimated from the DIBs is $E(B-V)=1.36 \pm 0.10$, where the error is the standard deviation of the averaged values. This colour excess agrees with that estimated from the photometric data. A B0V star has an intrinsic colour $(B-V)_{0}=-0.27$ (Wegner $1994)$ and taking the measured photometric colour $(B-V)=$ 1.10 we derive and excess $E(B-V)=1.37$. Likewise, assuming the interstellar extinction law $E(H-K)=0.20 E(B-V)$ and the intrinsic colour $(H-K)_{0}=-0.05$ for a main-sequence B0 star (Koornneef 1983) we obtain $E(B-V)=1.35$.

Finally, the distance can be estimated from the distancemodulus relation. Taking the standard law $A_{V}=3.1 E(B-$ $V)$ and assuming an average absolute magnitude for a B0V star of $M_{V}=-4.2$ (Vacca et al. 1996) the distance to SAX J2103.5+4545 is estimated to be $\sim 6.5 \pm 0.9 \mathrm{kpc}$. This error includes the errors in $m_{V}(0.02)$ and $A_{V}(0.3)$, but assumes no error in the absolute magnitude $M_{V}$. 

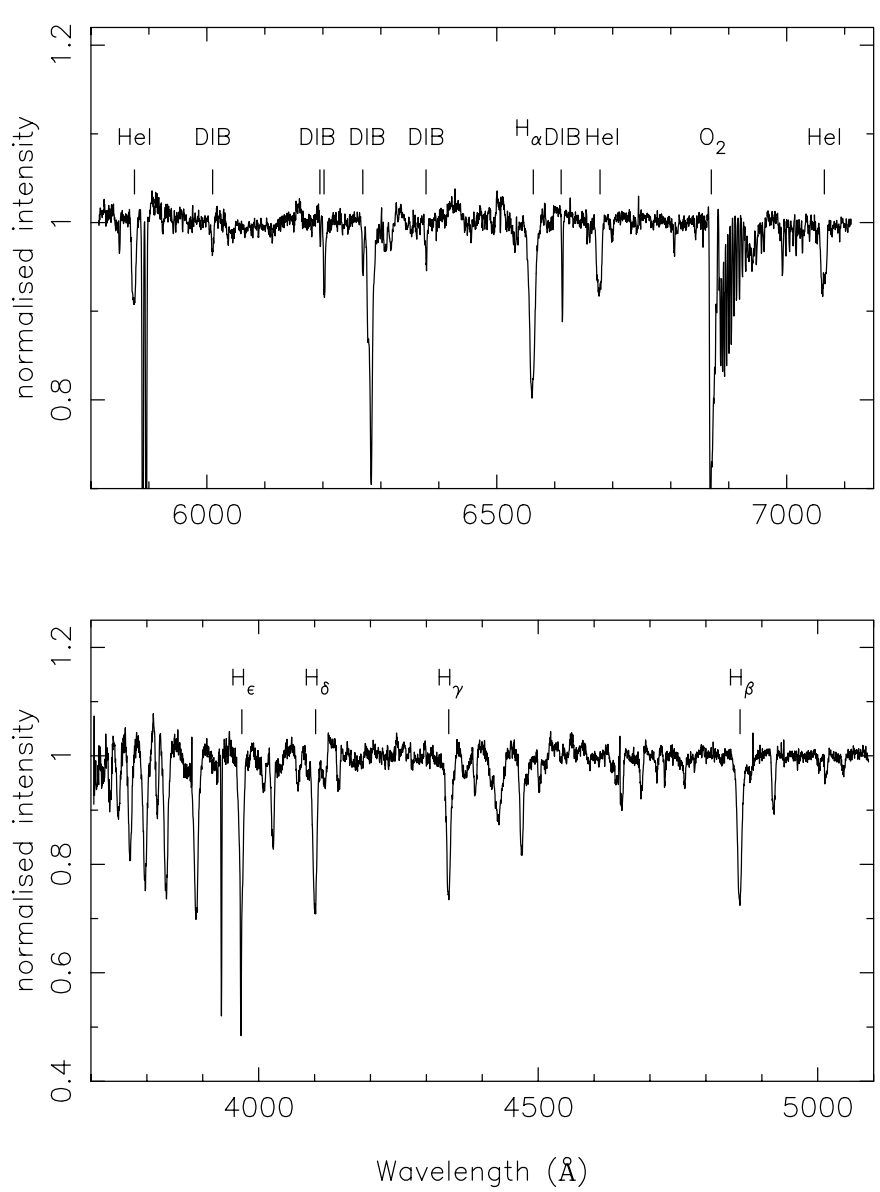

Fig. 5. Blue- and red-end spectra of the optical companion of SAX J2103.5+4545. We have indicated the diffuse interstellar bands used for the derivation of the reddening and the Helium lines $\lambda 5875$, 16678, $\lambda 7065$. The Balmer series up to $H_{13}$ can be seen. The spectra were taken from the WHT on September 14, 2003.

\subsection{Rotational velocity}

Be stars are fast rotators. They have, on average, larger observed rotational velocities than B stars as a group (Slettebak 1982). The determination of the rotational velocity is important because it has been seen to correlate with emission characteristics (Briot 1986; Mennickent et al. 1994). The rotational velocity can be estimated by measuring the full width at half maximum of $\mathrm{He} \mathrm{I}$ lines (e.g., Steele et al. 1999). We obtain $v \sin i=240 \pm 20 \mathrm{~km} \mathrm{~s}^{-1}$, which compares to the value of $246 \pm 16 \mathrm{~km} \mathrm{~s}^{-1}$ given for weak-emission early-type shell stars (Mennickent et al. 1994). As a comparison other rotational velocities in Be/X-ray binaries are: $v \sin i=200 \pm$ $30 \mathrm{~km} \mathrm{~s}^{-1}$ in LS I +61 235/RX J0146.9+6121 (Reig et al. 1997), $v \sin i=290 \pm 50 \mathrm{~km} \mathrm{~s}^{-1}$ in V635 Cas $/ 4 \mathrm{U} 0115+63$ (Negueruela \& Okazaki 2001), $v \sin i=240 \pm 20 \mathrm{~km} \mathrm{~s}^{-1}$ in LS 992/RX J0812.4-3114 (Reig et al. 2001).

\section{Discussion}

\subsection{SAX J2103.5+4545: A new Be/X-ray binary}

The two most significant observational characteristics of Be stars in the optical/infrared band are the emission lines and
Table 3. Diffuse interstellar lines used to estimate the reddening to SAX J2103.5+4545.

\begin{tabular}{cccc}
\hline \hline Line & $\begin{array}{c}E W^{a}(\mathrm{~m} \AA) \\
14 / 09 / 03\end{array}$ & $\begin{array}{c}E W(\mathrm{~m} \AA) \\
17 / 08 / 03\end{array}$ & $E(B-V)$ \\
\hline 6010 & 213 & - & - \\
6195 & 76 & 100 & $1.38 \pm 0.06$ \\
6202 & 341 & 300 & $1.42 \pm 0.18$ \\
6269 & 202 & 215 & $1.27 \pm 0.21$ \\
$6376 / 79$ & 193 & 215 & $1.24 \pm 0.07$ \\
6613 & 310 & 318 & $1.49 \pm 0.13$ \\
\hline Average & & $1.36 \pm 0.10$ \\
\hline & &
\end{tabular}

the infrared excess, compared with non line-emitting B stars of the same spectral type. These characteristics are associated with an equatorial outflow of material expelled from the rapidly rotating Be star that forms a quasi-Keplerian disc, whose effective temperature is $0.5-0.8$ cooler than that of the photosphere (Millar \& Marlborough 1999). Consequently, Be stars tend to appear redder than indicated by their spectral type. The slightly bluer colours of the source measured in August with respect to the June run and the progressive decrease of the $\mathrm{H} \alpha$ line intensity agrees with a weakening of the disc. Likewise, the rapid and large amplitude of variability of the infrared and $\mathrm{H} \alpha$ intensities indicate a perturbed disc. Nevertheless, the excellent agreement between the colour excess derived from the photometry and from the strength of interstellar lines (which are not affected by the circumstellar emission) indicates that the amount of material in the disc must be scarce during our observations.

The absorption derived from the X-ray data is, however, considerably higher than that derived from the optical observations. The value of the hydrogen column density measured by the X-ray missions ranges between $(3.1-3.8) \times 10^{22} \mathrm{~cm}^{-2}$ (Hulleman et al. 1998; Baykal et al. 2002), which represents $E(B-V) \approx 6$ (Predehl \& Schmitt 1995) or $E(B-V) \approx 5$ (Ryter et al. 1975; Gorenstein 1975), assuming $E(B-V)=$ $A_{V} / 3.1$ (Rieke \& Lebofsky 1985). This discrepancy can be explained by the different activity state of the source during the X-ray and optical observations. While the X-ray observations of Hulleman et al. (1998) and Baykal et al. (2002) took place during outbursts, the optical observations were carried out when the source was weakly X-ray active - according to contemporaneous RXTE/ASM measurements (see also Stark et al. 2003; Blay et al. 2004). In the Be/X-ray paradigm the high-energy radiation is consequence of accretion from the circumstellar disc. Thus during an outburst large amounts of material from the optical companion are expected. The detection of an iron line at $\sim 6.4 \mathrm{keV}$ supports the presence of circumstellar material. In contrast, no or small amounts of matter are accreted during low-activity X-ray states.

SAX J2103.5+4545 displays the so-called type I outbursts, i.e., recurrent and moderate $\left(L_{X} \lesssim 10^{37} \mathrm{erg} \mathrm{s}^{-1}\right)$ increases of $\mathrm{X}$-ray intensity covering a small fraction of the orbit and modulated with the orbital period. This transient behaviour is typical of Be/X-ray binaries and has been reported in a number of 
Table 4. Comparison of times of emission-to-absorption reversal of SAX J2103.5+4545 with two other Be/X-ray systems.

\begin{tabular}{cc|cc|cc}
\hline \hline \multicolumn{2}{c|}{ SAX J2103.5+4545 } & \multicolumn{2}{|c|}{$4 \mathrm{U} 0115+63^{b}$} & \multicolumn{2}{c}{$\mathrm{X} \mathrm{Per}^{c}$} \\
MJD & $E W(\mathrm{H} \alpha)$ & $\mathrm{MJD}$ & $E W(\mathrm{H} \alpha)$ & $\mathrm{MJD}$ & $E W(\mathrm{H} \alpha)$ \\
& $(\AA)$ & & $(\AA)$ & & $(\AA)$ \\
\hline 52853 & -2.20 & 50481 & -3.6 & 47925 & -1.3 \\
52869 & -1.07 & 50647 & +1.3 & 47950 & -1.14 \\
52897 & +2.32 & 50767 & -0.4 & 48137 & +1.6 \\
& & & & 48194 & +2.7 \\
\hline
\end{tabular}

\footnotetext{
${ }^{a}$ This work.

${ }^{b}$ Negueruela et al. (2001).

${ }^{c}$ Clark et al. (2001).
}

systems (e.g., Bildsten et al. 1997). Considering the distance estimated above, $6.5 \mathrm{kpc}$, the X-ray luminosity at the peak of the outbursts is $\sim 3 \times 10^{36} \mathrm{erg} \mathrm{s}^{-1}$, typical of type I outbursts. With this value of the distance and Galactic coordinates $l=87.12, b=-0.7$, SAX J2103.5+4545 agrees with a location within the Perseus spiral arm (Russeil 2003).

Before our optical observations the ascription of SAX J2103.5+4545 to the group of Be/X-ray binaries was circumstantial and based on X-ray data only. Our spectroscopic observations in the spectral classification region exposed a B0V star with a remarkable richness in spectral variability. We conclude that SAX J2103.5+4545 belongs to the group of $\mathrm{Be} / \mathrm{X}$-ray binaries.

\subsection{Disc truncation}

Figure 4 shows the profile of the $\mathrm{H} \alpha$ line in SAX J2103.5+4545. The corresponding equivalent widths are given in Table 2. The reversal of the $\mathrm{H} \alpha$ line from emission to absorption is one of the most spectacular types of variability among Be stars. It is interpreted as the loss of the circumstellar disc. Disc-loss episodes have been seen in a number of Be/X-ray binaries: X-per (Roche et al. 1993), A0 535+26 (Negueruela et al. 2000), RX J0812.4-3114 (Reig et al. 2001), 4U 0115+63 (Negueruela et al. 2001). During these episodes the strength of the $\mathrm{H} \alpha$ line and the photometric magnitudes and colours gradually (on time scales of months to years) fade away until the disc is lost. In SAX J2103.5+4545, these changes seem to be extremely fast. Although in order to fully appreciate the time scales for disc loss one should look at the long-term $\mathrm{H} \alpha$ variability, it is instructive to compare the time scales of the last instances of the emission-to-absorption reversal of SAX J2103.5+4545 with those of 4U0115+63 and X Per (Negueruela et al. 2001; Clark et al. 2001), as shown in Table 4. For RX J0812.4-3114 and A0 535+26 the observation are too sparse and do not allow us to constrain the time scales. For a similar change in the equivalent width of the $\mathrm{H} \alpha$ line, SAX J2103.5+4545 requires approximately 3 and 5 times less time than $4 \mathrm{U} 0115+63$ and X Per, respectively.

Given the small orbit and moderate eccentricity of the system disc truncation by the neutron star must have an important effect on the structure of the disc. According to the viscous decretion disc model, disc truncation is more effective in systems with low eccentricity $(e<0.2)$ than in systems with high eccentricity $(e>0.6)$ owing to their wider gap between the outer disc radius and the critical Roche lobe radius (Okazaki \& Negueruela 2001; Negueruela \& Okazaki 2001). SAX J2103.5+4545, has the smallest orbit among accretionpowered $\mathrm{Be} / \mathrm{X}$-ray binaries and is moderately eccentric $(e \sim$ $0.4)$. The combination of these two factors results in rather efficient disc truncation but also in small gaps that allow transfer of matter. Since mass transfer in $\mathrm{Be} / \mathrm{X}$-ray binaries occurs via the $L_{1}$ point of the critical Roche lobes, some amount of matter will be captured by the neutron star, hence producing outbursts.

Assuming typical values of the mass and radius of a B0V star $\left(M_{*}=20 M_{\odot}\right.$ and $R_{*}=8 R_{\odot}$, Vacca et al. 1996) and taking $e=0.4$, the periastron distance is estimated in $\sim 38 R_{\odot}$ or $\sim 5 R_{*}$. An estimate of the critical lobe radius yields virtually the same values. Thus, the neutron star acts very efficiently in removing angular momentum from the disc and preventing its growth. Another consequence for systems with small orbits is the absence of type II outburst - major increases in intensity, $L_{\mathrm{X}} \gtrsim 10^{37} \mathrm{erg} \mathrm{s}^{-1}$, that last for weeks or months and are usually accompanied by strong spin-up episodes of the neutron starsince the star does not have the opportunity of developing an extended and steady disc.

On the other hand, it is possible that a proper circumstellar disc never forms. A scenario like that seen in $\mu$ Cen (Baade et al. 1988; Hanuschik et al. 1993), where fast (2-5 days) ejections of photospheric material are lifted into bound orbits forming a short-lived ( $\$ 100$ days) quasi-stable Keplerian disc might be applicable to SAX J2103.5+4545 as well. $\mathrm{H} \alpha$ outbursts of this type are never very intense, the equivalent width does not go over a few $\AA$ and are accompanied by $V / R$ variability. Disc radii in such scenario do not go over 4-6 stellar radii (Hanuschik et al. 1993). The long periods of X-ray activity in 1997 and 1999/2000 would, however, argue against this scenario.

\subsection{The spin period-orbital period correlation}

The available data clearly demonstrate that SAX J2103.5+4545 is a Be/X-ray binary: i) in the X-ray band it appears as a moderately eccentric transient system that shows type I outbursts, ii) it shows X-ray pulsations and the X-ray spectral parameters are consistent with those found in other Be/X-ray systems, iii) the optical/IR counterpart is a B0Ve star that shows highly variable $\mathrm{H} \alpha$ emission. Still, if the 12.7 day modulation indeed represents the orbital period, then SAX J2103.5+4545 is the accretion-powered Be/X-ray binary with the shortest orbital period.

It is a well known fact that the two types of high-mass X-ray binaries (HMXB), Be/X, and supergiant systems (SXRB) occupy well-defined positions in the spin period versus orbital period diagram (Corbet 1986). Figure 6 displays an updated version of Corbet's diagram. SAX J2103.5+4545 deviates from the correlation of $\mathrm{Be} / \mathrm{X}$ systems and falls in the region of windfed supergiant X-ray binaries. The correlation in $\mathrm{Be} / \mathrm{X}$ systems is explained in terms of the equilibrium period, defined as the 


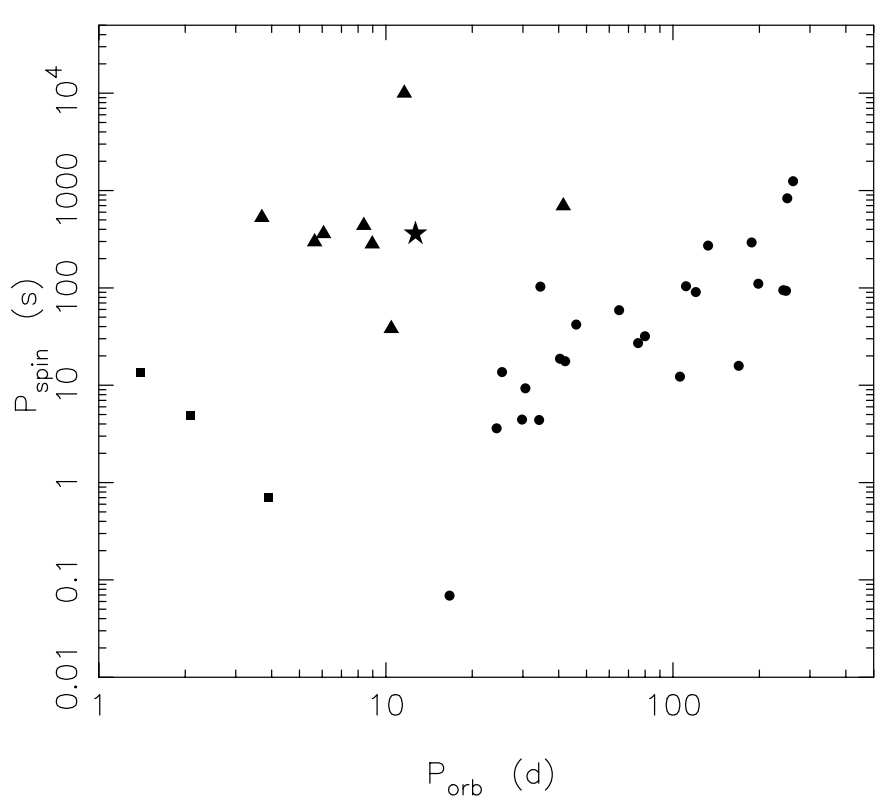

Fig. 6. $P_{\text {spin }}-P_{\text {orb }}$ diagram. The star symbol marks the position of SAX J2103.5+4545. Circles represent Be/X-ray binaries, triangles identify wind-fed supergiants and squares refer to disc-fed (Rochelobe filling) systems.

period at which the outer edge of the magnetosphere rotates with the Keplerian velocity (Davidson \& Ostriker 1973; Stella et al. 1986; Waters \& van Kerkwijk 1989).

According to Waters \& van Kerwijk (1989), wind-fed SXRB rotate at the value of the equilibrium period that they had when they were main-sequence stars. The subsequent winddriven accretion torques were not strong enough to spin up the neutron star after the spin-down that followed the supernova explosion. In Be/X-ray binaries, the slow spin-down that followed the supernova explosion was compensated by rapid spinup episodes due to the much more efficient ways of transfer angular momentum to the neutron star through the much higher density equatorial winds. If this interpretation is correct, then a Be/X-ray system going through a long period of quiescence can spin down to the equilibrium period that corresponds to the wind-driven accretion mechanism. In other words, in a quiescent $\mathrm{Be} / \mathrm{X}$-ray binary (presumably due to the lack of circumstellar matter) accretion takes place through the normal wind of an early-type star. Therefore, the neutron star would rotate at a rate more appropriate of a SXRB than of an active $\mathrm{Be} / \mathrm{X}$.

The spin-up rate of SAX J2103.5+4545 during the first $\sim 30$ days of the 1999 outburst was $2.5 \times 10^{-13} \mathrm{~Hz} \mathrm{~s}^{-1}$ (Baykal et al. 2000). This value is comparable to those observed in other Be/X-ray binaries during type I outbursts, where typical spin-up rates $\lesssim 5 \times 10^{-12} \mathrm{~Hz} \mathrm{~s}^{-1}$ have been reported (Wilson et al. 1997, 2002; Finger et al. 1996, 1999). However, the episodic spin-up phases in SAX J2103.5+4545 are not capable to exert strong enough torques on the neutron star to make it rotate at the equilibrium period. A more efficient way to spin up the neutron star would be through type II outbursts, for which typical values are $\gtrsim 8 \times 10^{-12} \mathrm{~Hz} \mathrm{~s}^{-1}$ (Bildsten et al. 1997, and references therein). These kind of outbursts, however, require high mass accretion rates that can only be achieved with a large and extended or dense disc. As explained above, due to the disc truncation SAX J2103.5+4545 can never develop such a disc.

In this respect, it is instructive to compared SAX J2103.5+4545 with the Be/X-ray system with the second shortest orbital period, A0538-66. In contrast to SAX J2103.5+4545 a spin period of only $66 \mathrm{~ms}$ has been reported for A0538-66 (Skinner et al. 1982), which would be indicative of large amounts of angular momentum transfer. In the early 80's A0538-66 experienced a series of very luminous (super-Eddington) outbursts regularly separated by $16.7 \mathrm{~d}$, which was interpreted as the orbital period. In the standard model for this source (Charles et al. 1983), it is believed that the eccentricity of the neutron star's orbit is very high, so that the neutron star comes very close to the surface of the B-type star at periastron, sometimes inducing moderate Roche-lobe overflow. In addition, A0538-66 is extremely active in the optical band (Alcock et al. 2001) and high accretion rates must be sustained. Hence, we can suspect that spin-up episodes must be occurring frequently in A0538-66.

\section{Conclusions}

We have identified the optical counterpart to the X-ray transient SAX J2103.5+4545. The optical spectroscopic observations revealed a B0Ve star displaying fast spectral changes. Due to the short orbital period and moderate eccentricity, the neutron star truncates the Be star's disc at a small radius and prevents the development of an extended and steady disc. The impossibility to develop a dense disc implies that most of the time the density and velocity profiles of the stellar wind, i.e, of the accreted material, are those of a normal B star rather than the much slower and denser wind of a Be star. The result is a slow neutron star that rotates far away from its equilibrium period. The equatorial disc formed under these conditions is short-lived and perturbed, having density gradients that lead to the formation of asymmetries that translate into $V / R$ variability. SAX J2103.5+4545 is an excellent example of how the neutron star affects the physical properties of the Be star companion.

Acknowledgements. P.R. and I.N. are researchers of the programme Ramón y Cajal funded by the Spanish Ministery of Science and Technology and the Universities of Valencia and Alicante, under grants ESP2002-04124-C03-01 and ESP2002-04124-C03-03, respectively. P.R. acknowledges partial support by the Agencia Velenciana de Ciencia y Tecnología under grant CTESPP/2003/002. Skinakas Observatory is a collaborative project of the University of Crete, the Foundation for Research and Technology-Hellas and the Max-PlanckInstitut für Extraterrestrische Physik. Some of the WHT spectra were obtained as part of the ING service programme.

\section{References}

Alcock, C., Allsman, R. A., Alves, D. R., et al. 2001, MNRAS, 321, 678

Baade, D., Dachs, J., van de Weygaert, R., \& Steeman, F. 1988, A\&A, 198, 211

Baykal, A., Stark, M. J., \& Swank, J. H. 2000, ApJ, 544, 129

Baykal, A., Stark, M. J., \& Swank, J. H. 2002, ApJ, 569, 903 
Bildsten, L., Chakrabarty, D., Chiu, J., et al. 1997, ApJS, 113, 367

Blay, P., Reig, P., Martínez, S., et al. 2004, A\&A, submitted

Briot, D. 1986, A\&A, 163, 67

Charles, P. A., Booth, L., Densham, R. H., et al. 1983, MNRAS, 202, 657

Clark, J. S., Tarasov, A. E., Okazaki, A. T., Roche, P., \& Lyuty, V. M. 2001, A\&A, 380, 615

Corbet, R. H. D. 1986, MNRAS, 220, 1047

Davidson, K., \& Ostriker, J. P. 1973, ApJ, 179, 585

Finger, M. H., Wilson, R. B., \& Chakrabarty, D. 1996, A\&AS, 120, 209

Finger, M. H., Bildsten, L., Chakrabarty, D., et al. 1999, ApJ, 517, 449

Galazutdinov, G. A., Musaev, F. A., Krelowski, J., \& Walker, G. A. H. 2000, PASP, 112, 648

Gorenstein, P. 1975, ApJ, 198, 95

Grebel, E. K. 1997, A\&A, 317, 448

Hanuschik, R. W., Dachs, J., Baudzus, M., \& Thimm, G. 1993, A\&A, 274,356

Herbig, G. H. 1975, ApJ, 196, 129

Herbig, G. H., \& Leka, K. D. 1991, ApJ, 382, 193

Howarth, I. D., Murray, J., Mills, D., \& Berry, D. S. 1998, Starlink User Note 50.21

Hulleman, F., in 't Zand, J. J. M., \& Heise, J. 1998, A\&A, 337, L25

Hunt, L. K., Mannucci, F., \& Testi, L., et al. 1998, AJ, 115, 2594

Inam, S. C., Baykal, A., Swank, J., \& Stark, M. J. 2004, ApJ, submitted [arXiv: astro-ph/0402221]

Jaschek, C., \& Jaschek, M. 1987, in The classification of the Stars (Cambridge University Press)

Koornneef, J. 1983, A\&A, 128, 84

Kothes, R., Reich, W., Foster, T., \& Byun, D. 2003, ApJ, 588, 852

Mennickent, R. E., Vogt, N., Barrera, L. H., Covarrubias, R., \& Ramirez, A. 1994, A\&AS, 106, 427

Millar, C. E., \& Marlborough, J. M. 1999, ApJ, 526, 400
Negueruela, I., Reig, P., Finger, M. H., \& Roche, P. 2000, A\&A, 356, 1003

Negueruela, I., \& Okazaki, A. T. 2001, A\&A, 369, 108

Negueruela, I., Okazaki, A. T., Fabregat, J., et al. 2001, A\&A, 369, 117

Landolt, A. U. 1992, AJ, 104, 340

Okazaki, A. T., \& Negueruela, I. 2001, A\&A, 377, 161

Predehl, P., \& Schmitt, J. H. M. M. 1995, A\&A, 293, 889

Reig, P., Fabregat, J., Coe, M. J., et al. 1997, A\&A, 322, 183

Reig, P., Negueruela, I., Buckley, D. A. H., et al. 2001, A\&A, 367, 266

Rieke, G. H., \& Lebofsky, M. J. 1985, ApJ, 288, 618

Roche, P., Coe, M. J., Fabregat, J., et al. 1993, A\&A, 270, 122

Russeil, D. 2003, A\&A, 397, 133

Ryter, C., Cesarsky, C. J., \& Audouze, J. 1975, ApJ, 198, 103

Shortridge, K., Meyerdierks, H., Currie, M., et al. 2001, Starlink User Note 86.19

Skinner, G. K., Bedford, D. K., Elsner, R. F., et al. 1982, Nature, 297, 568

Slettebak, A. 1982, ApJS, 50, 55

Stella, L., White, N. E., \& Rosner, R. 1986, ApJ, 308, 669

Stark, M. J., Saia, M., Swank, J. H., \& Baykal, A. 2003, Am. Astron. Soc. Meet. 203, \#53.05

Steele, I. A., Negueruela, I., \& Clark, J. S. 1999, A\&AS, 137, 147

Stevens, J. B., Coe, M. J., \& Buckley, D. A. H. 1999, MNRAS, 309, 421

Vacca, W. D., Garmany, C. D., \& Shull, J. M. 1996, ApJ, 460, 914

Walborn, N. R., \& Fitzpatrick, E. L. 1990, PASP, 102, 379

Waters, L. B. F. M., \& van Kerkwijk, M. H. 1989, A\&A, 223, 196

Wegner, W. 1994, MNRAS, 270, 229

Wilson, C. A., Finger, M. H., Harmon, B. A., et al. 1997, ApJ, 479, 388

Wilson, C. A., Finger, M. H., Coe, M. J., Laycock, S., \& Fabregat, J. 2002, ApJ, 570, 287 